\title{
Hallucinations after Ingesting a High Dose of Benzydamine Hydrochloride
}

\author{
Burak $\mathrm{Can}^{1}$, Ihsan $\mathrm{Oz}^{2}$, Husameddin $\mathrm{Ozer}^{3}$, Turgay Simsek ${ }^{4}$ \\ Departments of ${ }^{1}$ Internal Medicine, ${ }^{2}$ Emergency Medicine, ${ }^{3}$ Psychiatry, and ${ }^{4}$ General Surgery, ToyotaSA Emergency Aid Hospital, Arifiye, Turkey
}

\section{TO THE EDITOR}

Benzydamine hydrochloride $(\mathrm{BH})$ is a nonsteroidal anti-inflammatory drug (NSAID) which acts locally. ${ }^{1)}$ There are several different preparations of benzydamine; capsule, mouthwash, dermal cream, preparations are aerosols and vaginal inserts. Side effects include urticaria, erythema, rash, photosensitivity, bronchospasm, and renal function disorders. ${ }^{2)}$ Suggested therapeutic doses are 0.7-1.0 mg/kg per oral. ${ }^{3)}$ Hallucination, agitation, seizures can occur with $\mathrm{BH}$ poisoning. ${ }^{4}$

Eighteen-year-old male patient who attempted suicide had swallowed 20 pieces of candy containing $50 \mathrm{mg} \mathrm{BH}$. An hour after swallowing the drugs the patient was brought to the emergency room by his family. He said he saw people walking in the emergency room. This was visual hallucination. At physical examination there was no abnormal finding except hallucinations. His previous history was otherwise unremarkable, and he did not report any psychiatric acute or chronic disease. He denied alcohol consumption. Blood pressure 130/80 $\mathrm{mmHg}$, pulse 80 beats/minutes, $\mathrm{SpO}_{2} 96 \%$, body temperature $36.6^{\circ} \mathrm{C}$ was detected. Electrocardiography was normal sinus rhythm, and there was no pathological findings in routine biochemical tests, blood gases, blood count. Patient was cosultated with National Poison Information Centre and activated charcoal administration and hospitalization was suggested. Patient was observed at intensive care unit. Then, patients admitted to the intensive care unit of our hospital were monitored. Oral intake was stopped and intravenous hydration started to protect kidney functions. Intravenous pantoprazole was given for possible gastric

\footnotetext{
Received: January 16, 2016 / Revised: January 29, 2016

Accepted: January 30, 2016

Address for correspondence: Burak Can, MD

Department of Internal Medicine, ToyotaSA Emergency Aid Hospital,

Kirazca Mah. Gişeler Mevki Tem Otoyolu, Arifiye, Sakarya

(Adapazar1), Turkey

Tel: +90-2642291600, Fax: +90-2642294319

E-mail: brk_cn@yahoo.com
}

side effects of benzydamine. Patient's hallucinations were ongoing 5 hours of hospitalization. He said he saw mices inside the intensive care unit. After 10 hours of hospitalization, hallucinations was totally resolved. Twenty-four hours later the patient received inpatient services. Any toxic effect did not developed so he was observed 3 days and then discharged.

$\mathrm{BH}$ abuse for the purpose of suicide is a rare condition. Recreational use of $\mathrm{BH}$, because of its hallucinogen effect, has been reported. ${ }^{2)}$ The mechanism of hallucination development of benzydamine is unknown.

Who abuses $\mathrm{BH}$ for recreatinal purposes usually take it with alcohol. It is difficult to distinguish cause of hallucination; benzydamine or alcohol. Also in patients with psychiatric disorders, psychiatric side effects can occur even at normal doses of $\mathrm{BH}$, and overdose can lead to chronic psychosis. ${ }^{5-7)}$ In this case, patients previous history was unremarkable, and he did not reported any psychiatric disorders. He denied alcohol consumption. So we thought that cause of hallucination directly $\mathrm{BH}$.

Benzydamine hydrochloride is a widely used drug. It can cause phsychotic adverse effects at normal dose or overdose. Physicians should be keep in mind the psychiatric side effects of $\mathrm{BH}$.

\section{REFERENCES}

1. Turnbull RS. Benzydamine Hydrochloride (Tantum) in the management of oral inflammatory conditions. $J$ Can Dent Assoc 1995;61:127-134.

2. Opaleye ES, Noto AR, Sanchez Zv, Moura YG, Galduróz JC, Carlini EA. Recreational use of benzydamine as a hallucinogen among street youth in Brazil. Rev Bras Psiquiatr 2009;31:208-213.

3. Silvestrini B, Barcellona PS, Garau A, Catanese B. Toxicology of benzydamine. Toxicol Appl Pharmacol 1967;10: 148-159.

4. Baldock GA, Brodie RR, Chasseaud LF, Taylor T, Walmsley LM, Catanese B. Pharmacokinetics of benzydamine after intravenous, oral, and topical doses to human subjects. Biopharm Drug Dispos 1991;12:481-492.

5. Doksat MK, Yilmaz C, Caksen H, Guven AS. Reversible worsening of psychosis due to benzydamine in a schizoaffective young girl who is already under treatment with

(a) This is an Open-Access article distributed under the terms of the Creative Commons Attribution Non-Commercial License (http://creativecommons.org/licenses/by-nc/4.0) which permits unrestricted non-commercial use, distribution, and reproduction in any medium, provided the original work is properly cited. 
antipsychotics. Bull Clin Psychopharmacol 2009;19:279284.

6. Guru M, Safak Y, Kuru E, Orsel S. Psychotic disorder related to benzydamine hydrochloride abuse: a case report. Bull Clin Psychopharmacol 2012;22 Suppl 1:S51.
7. Schifano F, Corazza O, Marchi A, Di Melchiorre G, Sferrazza $\mathrm{E}$, Enea A, et al; Psychonaut web mapping e rednet research projects. Analysis of online reports on the potential misuse of benzidamine. Riv Psichiatr 2013;48:182-186. 